Peritonitis, resulting from either malpositioning of the gastrostomy tube or leakage to the peritoneal cavity [1-3] is reported as a major complication following the percutaneous endoscopic gastrostomy (PEG) procedure, which may lead to lethal conditions including sepsis.

The aim of this study was to evaluate safety with regard to a novel step: a threepoint triangular fixation of the stomach to the abdominal wall in the PEG procedure (Fig. 1). We applied this three-point fixation, using a commercially available fixation kit, the "Funada-style" kit (TOP Corporation, Tokyo, Japan), in 31 patients with stroke or hypoxic encephalopathy, between November 2001 and October 2003 at Kanazawa Medical University Hospital.

The presence of complications following PEG was retrospectively analyzed by reviewing the hospital medical charts. The complication rate following the PEG with a triple gastropexy was compared with that following PEG without gastropexy $(n=10)$. No adverse events, including peritonitis or hemorrhage, were observed during the follow-up period after the PEG with three-point fixation, except for transient mild fever (less than $38 \mathrm{C}$ ) in two patients. On the other hand, subcutaneous abscess (complication rate $40 \%$ ) and gastric ulcer caused by the bumper on the mucosa $(20 \%)$ were observed in the patients who underwent PEG without gastropexy. Using the three-point fixation method, we were able to obtain a minimal distance $(30-40 \mathrm{~mm}$ ) between the stomach and the abdominal wall, and it appears that this mainly contributed to the lower occurrence of complications following PEG.

Our results suggest that three-point triangular fixation of the stomach to the abdominal wall in the PEG procedure might establish a safe route for tube feeding. Future studies with larger numbers and nonselected patient groups, and with

\title{
Three-Point Fixation of Stomach to Abdominal Wall in the Percutaneous Endoscopic Gastrostomy Procedure
}

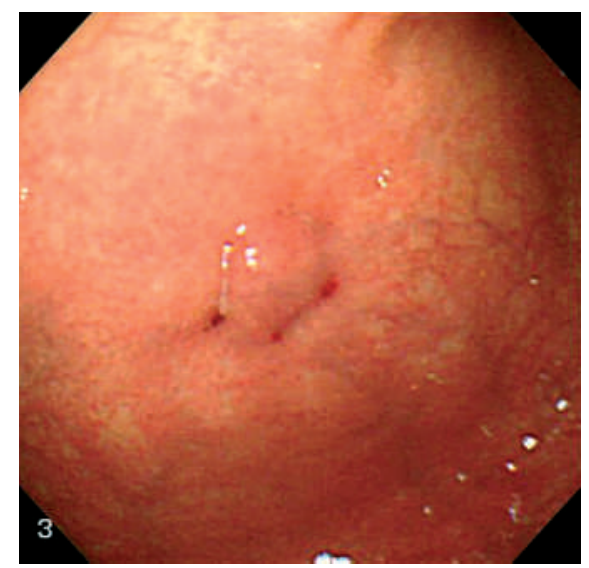

Figure 1 Three-point triangular fixation of stomach to abdominal wall with a commercially available fixation kit, in a percutaneous endoscopic gastrostomy (PEG) procedure (intragastric view).

long-term follow up, are needed to clarify the possible advantage of this method.

\section{K. Kusaka, T. Itoh, K. Kawaura,}

J. Yamakawa, T. Takahashi, T. Kanda

Department of General Medicine, Kana-

zawa Medical University, Ishikawa, Japan.

\section{References}

${ }^{1}$ Grant JP. Percutaneous endoscopic gastrostomy. Initial placement by single endoscopic technique and long-term follow-up. Ann Surg 1993; 217: 168 174

${ }^{2}$ Peteren TI, Kruse A. Complications of percutaneous endoscopic gastrostomy. Eur J Surg 1997; 163: 351 - 356

${ }^{3}$ Kimber CP, Khattak IU, Kiely EMet al. Peritonitis following percutaneous gastrostomy in children: management guidelines. Aust N Z J Surg 1998; 68: $268-270$

\section{Corresponding Author}

\section{T. Kanda, M.D.}

Department of General Medicine Kanazawa Medical University 1-1 Daigaku, Uchinada-machi Kahoku-gun Ishikawa 920-0293

Japan

Fax: $\quad$ +81-76-2862702

E-mail: kandat@kanazawa-med.ac.jp 